\title{
Space Launch System Spacecraft and Payload Elements: Making Progress Toward First Launch
}

\author{
Andrew A. Schorr ${ }^{1}$, Stephen D. Creech ${ }^{2}$, Michael Ogles ${ }^{3}$, and David Hitt ${ }^{4}$ \\ NASA Marshall Space Flight Center, Huntsville, AL, 35812
}

\begin{abstract}
Significant and substantial progress continues to be accomplished in the design, development, and testing of the Space Launch System (SLS), the most powerful humanrated launch vehicle the United States has ever undertaken. Designed to support human missions into deep space, SLS is one of three programs being managed by the National Aeronautics and Space Administration's (NASA's) Exploration Systems Development directorate. The Orion spacecraft program is developing a new crew vehicle that will support human missions beyond low Earth orbit, and the Ground Systems Development and Operations (GSDO) program is transforming Kennedy Space Center (KSC) into nextgeneration spaceport capable of supporting not only SLS but also multiple commercial users. Together, these systems will support human exploration missions into the proving ground of cislunar space and ultimately to Mars. SLS will deliver a near-term heavy-lift capability for the nation with its 70 metric ton (t) Block 1 configuration, and will then evolve to an ultimate capability of $130 \mathrm{t}$. The SLS program marked a major milestone with the successful completion of the Critical Design Review in which detailed designs were reviewed and subsequently approved for proceeding with full-scale production. This marks the first time an exploration class vehicle has passed that major milestone since the Saturn V vehicle launched astronauts in the 1960s during the Apollo program. Each element of the vehicle now has flight hardware in production in support of the initial flight of the SLS, Exploration Mission-1 (EM-1), an un-crewed mission to orbit the moon and return, and progress is on track to meet the initial targeted launch date in 2018. In Utah and Mississippi, booster and engine testing are verifying upgrades made to proven shuttle hardware. At Michoud Assembly Facility (MAF) in Louisiana, the world's largest spacecraft welding tool is producing tanks for the SLS core stage. This paper will particularly focus on work taking place at Marshall Space Flight Center (MSFC) and United Launch Alliance (ULA) in Alabama, where upper stage and adapter elements of the vehicle are being constructed and tested. Providing the Orion crew capsule/launch vehicle interface and in-space propulsion via a cryogenic upper stage, the Spacecraft/Payload Integration and Evolution (SPIE) Element serves a key role in achieving SLS goals and objectives. The SPIE element marked a major milestone in 2014 with the first flight of original SLS hardware, the Orion Stage Adapter (OSA) which was used on Exploration Flight Test-1 with a design that will be used again on EM-1. Construction is already underway on the EM-1 Interim Cryogenic Propulsion Stage (ICPS), an in-space stage derived from the Delta Cryogenic Second Stage. Manufacture of the Orion Stage Adapter and the Launch Vehicle Stage Adapter is set to begin at the Friction Stir Facility located at MSFC while structural test articles are either completed (OSA) or nearing completion (Launch Vehicle Stage Adapter). An overview is provided of the launch vehicle capabilities, with a specific focus on SPIE Element qualification/testing progress, as well as efforts to provide access to deep space regions currently not available to the science community through a secondary payload capability utilizing CubeSat-class satellites.
\end{abstract}

\footnotetext{
${ }^{1}$ Assistant Manager (Acting), Spacecraft/Payload Integration \& Evolution, XP50, Non-Member.

${ }^{2}$ Deputy Manager, Spacecraft/Payload Integration \& Evolution, XP50, Non-Member.

${ }^{3}$ Director of NASA Programs, Samuel Ginn College of Engineering, Auburn University, Associate Member

${ }^{4}$ Communication Strategist, Program Integration Office, XP02, AIAA Member.
} 


\section{Introduction}

$\mathrm{C}$ reated to provide sufficient launch capability to enable human exploration missions beyond Earth orbit and ultimately to Mars, NASA's Space Launch System rocket represents a new asset, not only for human spaceflight, but also for a variety of other payloads and missions with launch requirements beyond what is currently available. The initial configuration of the vehicle, on track for launch readiness in 2018, is designed to offer substantial launch capability in an expeditious timeframe and to support evolution into configurations offering greater launch capability via an affordable and sustainable development path.

NASA is developing SLS in parallel with two other exploration systems development efforts - the Orion crew vehicle program and the Ground Systems Development and Operations program. Orion is designed to carry astronauts on exploration missions into deep space. GSDO is converting the facilities at NASA's Kennedy Space Center in Florida into a nextgeneration spaceport capable of supporting launches by multiple types of vehicles.

These capabilities are part of a larger NASA strategy of working with commercial partners that will support crew and cargo launches to the International Space Station, while the agency focuses its development efforts on an incremental approach to developing the systems necessary for human

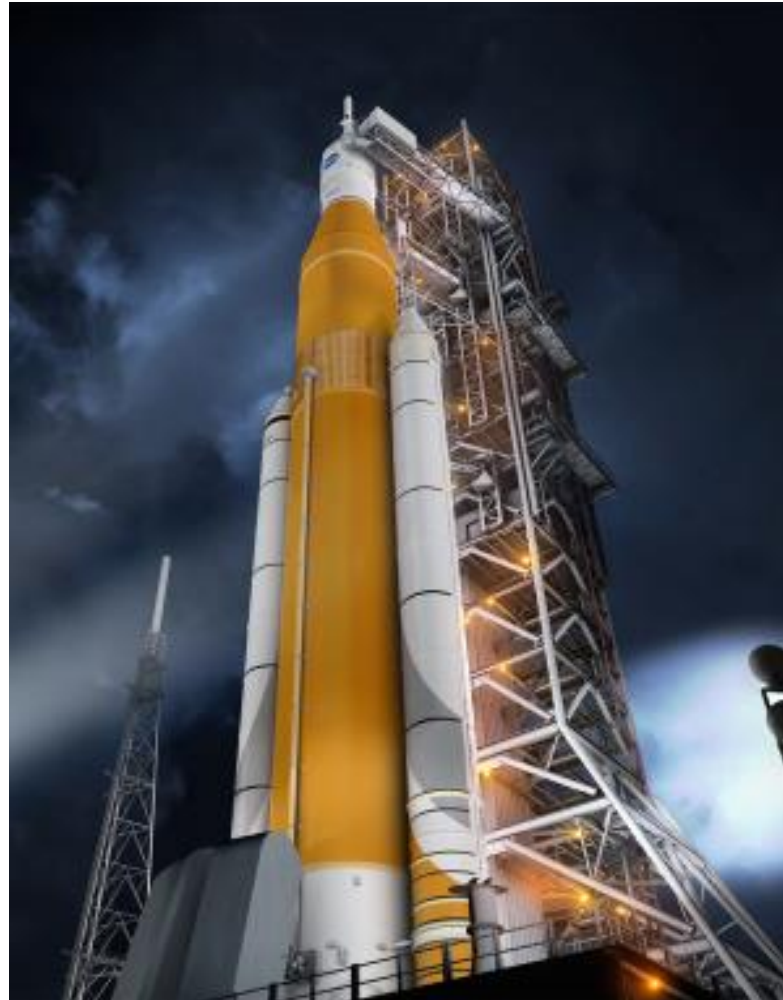

Figure 1. Artist's rendition of Space Launch System on its mobile launcher. exploration beyond Earth orbit and eventually to Mars.

SLS is being designed with performance margin and flexibility to support an evolvable human exploration approach.

Currently under construction, the initial configuration of the vehicle (Figure 1) will have the capability to deliver a minimum of $70 \mathrm{t}$ into low Earth orbit (LEO) and launch a crew aboard the Orion spacecraft into cislunar space on its first flight in 2018. The vehicle will evolve to a full capability of greater than $130 \mathrm{t}$ to LEO and will be able to support a stepping-stone approach to human exploration leading to the first footsteps on Mars.

\section{Vehicle Overview and Status}

The architecture of the SLS initial Block 1 configuration reflects NASA's desire to meet the mandates for heavylift capability in the U.S. congressional NASA Authorization Act of 2010 in a manner that is safe, affordable, and sustainable. After input was received from industry and numerous concepts were reviewed, a shuttle-derived design was found to enable the safest, most-capable transportation system in the shortest amount of time for the anticipated near-term and long-range budgets.

The SLS operational scheme takes advantage of resources established for the Space Shuttle Program, including workforce, tooling, manufacturing processes, supply chains, transportation logistics, launch infrastructure, and liquid oxygen and hydrogen (LOX/LH2) propellants and allows the initial configuration of the vehicle to be delivered with only one clean-sheet new development, the Core Stage. In October 2015, the SLS Program completed its Critical Design Review (CDR), the first time a NASA human-class launch vehicle has reached that milestone since the Shuttle Program almost 40 years ago and the first for an exploration-class vehicle since the Saturn V. 
The SLS Core Stage, which stores the liquid oxygen (LOX) and liquid hydrogen (LH2) propellant for four Core Stage engines, represents almost two-thirds of the vehicle's 97-meter height, standing $61 \mathrm{~m}$ tall, and will have a diameter of $8.4 \mathrm{~m}$, sharing commonality with the space shuttle's external tank in order to enhance compatibility with equipment and facilities at KSC and elsewhere. At MAF, outside New Orleans, Louisiana, the world's largest space vehicle welding tool, the $52 \mathrm{~m}$-tall Vertical Assembly Center (VAC), is currently being used by The Boeing Company, Core Stage prime contractor, to weld barrel sections, rings and domes together to form the test and flight articles of the propellant tanks for the stage. (Figure 2)

The Core Stage will be powered by four RS-25 engines, which previously served as the Space Shuttle Main Engine (SSME), taking advantage of 30 years of U.S. experience with liquid oxygen and liquid hydrogen, as well as an existing U.S. national infrastructure that includes specialized manufacturing and launching facilities. These human-rated engines support the SLS pursuit of safety, with a record of 100 percent mission success for the engines over 135 flights. At the end of the Space Shuttle Program, 16 RS-25 flight engines and two development engines were transferred to the SLS Program and placed in inventory at NASA's Stennis Space Center, providing enough engines for the first four flights of SLS.

Modifications to Stennis Test Stand A-1 to support RS25 testing were completed in 2014, and testing has been underway since the beginning of 2015 in preparation for flight certification of the SLS configuration of the engine, including a new engine controller unit (Figure 3). The testing includes propellant pressure and temperature inlet conditions that will both be higher with SLS than with the shuttle, as well as other SLS-specific performance requirements such as 109 percent thrust versus the shuttle's 104.5 percent thrust. Stennis Test Stand B-2 is being refitted for the SLS "green run" - the test firing of the first Core Stage with four RS-25 engines beginning in late 2017, which will be NASA's largest liquid engine ground firing since stage tests of the Saturn V in the 1960s.

The majority of the thrust for the first two minutes of flight will come from a pair of Solid Rocket Boosters, also of Space Shuttle Program heritage. The SLS is upgrading the boosters from the four-segment version flown on the shuttle to a more-powerful five-segment version. Each booster measures $54 \mathrm{~m}$ long and $3.7 \mathrm{~m}$ in diameter and is capable of generating up to 3.6 million pounds of thrust, the most powerful flight boosters in the world. Although largely similar to the SRBs used on the space shuttle, this upgraded five-segment SRB includes improvements such as a larger nozzle throat and an environmentally-benign insulation and liner material (asbestos-free). In June 2016, the SLS configuration of the booster successfully

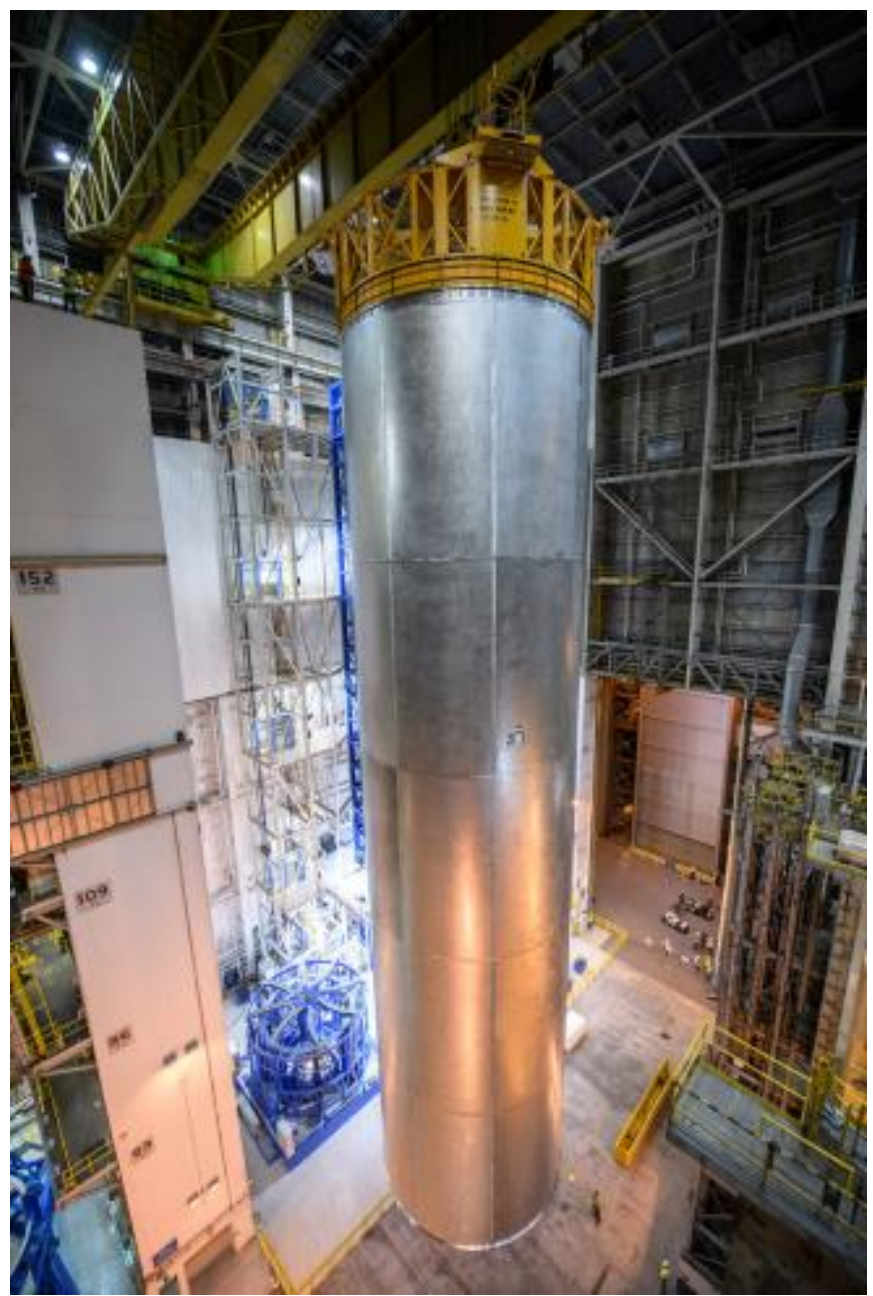

Figure 2. Production of the structural test article of the liquid hydrogen tank for the SLS Core Stage.

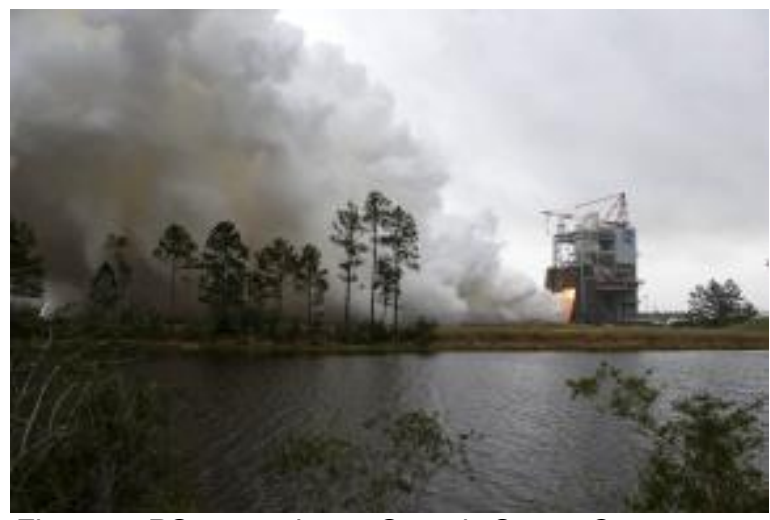

Figure 3. RS-25 testing at Stennis Space Center. 
underwent the second of two Qualification Motor tests, and booster hardware is currently being prepared for first flight. (Figure 4)

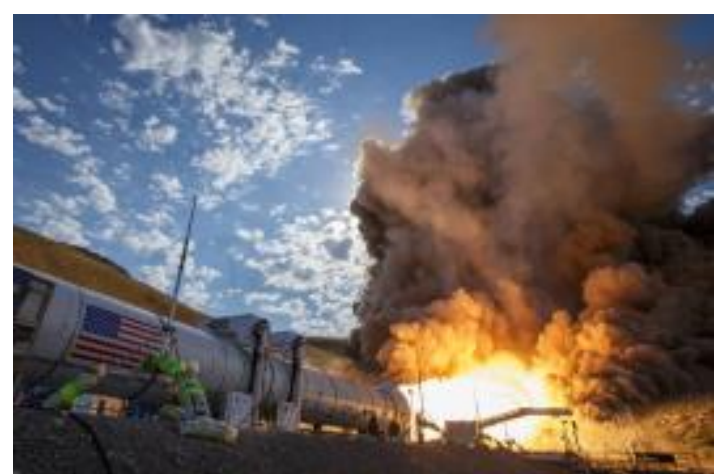

Figure 4. Qualification Motor-2 test of the SLS solid rocket boosters.

\section{Spacecraft/Payload Element Development}

One of four hardware element offices of the SLS Program, the SPIE Office is responsible for the analysis, design, development, and/or procurement of the SLS payload and vehicle adapters and for payload fairings and fairing adapters, including launch vehicle-to-spacecraft and launch vehicle-to-payload accommodations, interfaces, and integration. In addition the Office is responsible for procuring and modifying the ICPS for in-space propulsion of the EM-1 mission specifically.

In order to expedite earlier initial launch of this new U.S. super-heavy-lift launch capability, the decision was made early in the vehicle's development to leverage the proven Delta Cryogenic Second Stage (DCSS) for SLS inspace propulsion, delaying development of a larger upper stage until the vehicle's Core Stage, the largest new development for the Block 1 vehicle, was more mature. That decision has allowed the program to move toward initial launch readiness, with the capability to send the Orion crew vehicle farther into space than Apollo travelled. The Block 1 Spacecraft/Payload Elements include not only the ULA-produced DCSS-derived ICPS but also two adapters, connecting the stage to the Core Stage and to the Orion spacecraft. (Figure 5)

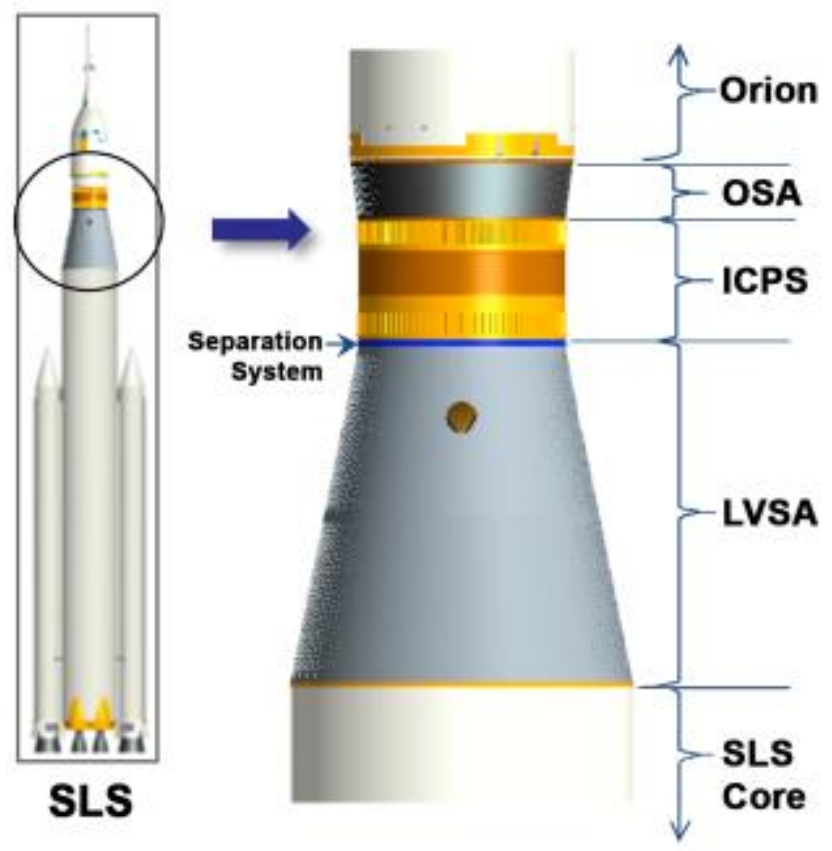

Figure 5. Spacecraft Payload Elements.

4

American Institute of Aeronautics and Astronautics 


\section{A. Launch Vehicle Stage Adapter (LVSA)}

Representing the primary interface component to Stage, the LVSA serves multiple roles as an adapter from the Core Stage to the Hydrogen Tank of the ICPS, serving as a primary load path structure, providing the Separation Plane via a Frangible Joint Assembly (FJA) and Pneumatic Actuation System (PAS) currently utilized on the ULA Delta and Atlas launch vehicles (Figure 6). In addition, the LVSA incorporates accommodations to route communication cabling as well as attachment points for technician platforms to allow launch preparation and closeout activities on the ICPS hardware that is nested inside the adapter housing. Approximate dimensions of the lightweight aluminum cone are $5 \mathrm{~m}(\sim 16.5 \mathrm{ft})$ and $8.4 \mathrm{~m}(\sim 27.5 \mathrm{ft})$ for the forward and aft circumferences respectively while standing.

Prime contractor responsible for the Design, Development, Test, and Evaluation (DDT\&E) and final Qualification is Teledyne Brown Engineering (TBE) based in Huntsville, AL. Actual manufacturing of the adapter was performed by Marshall Spaceflight Center (MSFC) utilizing Friction Stir Welding (FSW) capability (traditional FSW Vertical Weld Tool and Self-Reacting FSW Robotic Weld Tool) resident at MSFC under a Space Act Agreement to TBE.

Specific design aspects of the LVSA are noted in Figure 7. The lower bolted interface provides attachment capability to the Core Stage, while the forward joint incorporates the FJA/PAS in addition to enabling the interface to the Hydrogen fuel tank. Two (2) circular doors forward and two (2) circular doors aft provide the necessary access for launch site technicians to install removable platforms for required pre-launch and if-needed, offnominal operations. Passive vents are located in the aft cone to prevent the structure from generating a delta pressure that could potentially either result in a burst or crush (buckling) condition that compromises the structural integrity of the hardware.

Panels of lightweight Aluminum are machined, formed, and heat-treated prior to delivery to MSFC for welding. In addition to the overall dimensional machining performed, the interior surfaces of the panels are machined to a lattice-like orthogrid configuration to provide the necessary structural integrity while reducing weight.

A unique aspect of the manufacturing approach was the need to manufacture the adapter in two (2) cones, forward and aft. This was required primary due to one (1) piece panels meeting the height requirement in the lightweight alloy utilized was unavailable within current industry capability. The decision then became whether to use a bolted or welded joining technique to combine the two (2) cones. In the interest of weight savings, it was decided that a FSW joining of the cones would be preferred approach. However, the challenge then became how to securely hold the adjacent cones together in order to complete the FSW mating of the cones. Through the innovative application of a modular tooling approach and utilization of Hawthorne clamps developed during the Saturn Launch Vehicle Program, the cones were successfully mated with no issue. Substantial cost and schedule savings were realized versus a more traditional exoskeleton tooling approach.

To date, the Structural Test Article unit has completed manufacture and is currently undergoing installation of necessary instrumentation for the gathering of required data from the test. Once completed, the LVSA will be transported to the MSFC Test Area to be mated to the Core Stage Simulator and subsequently to the Hydrogen Tank of the ICPS. 


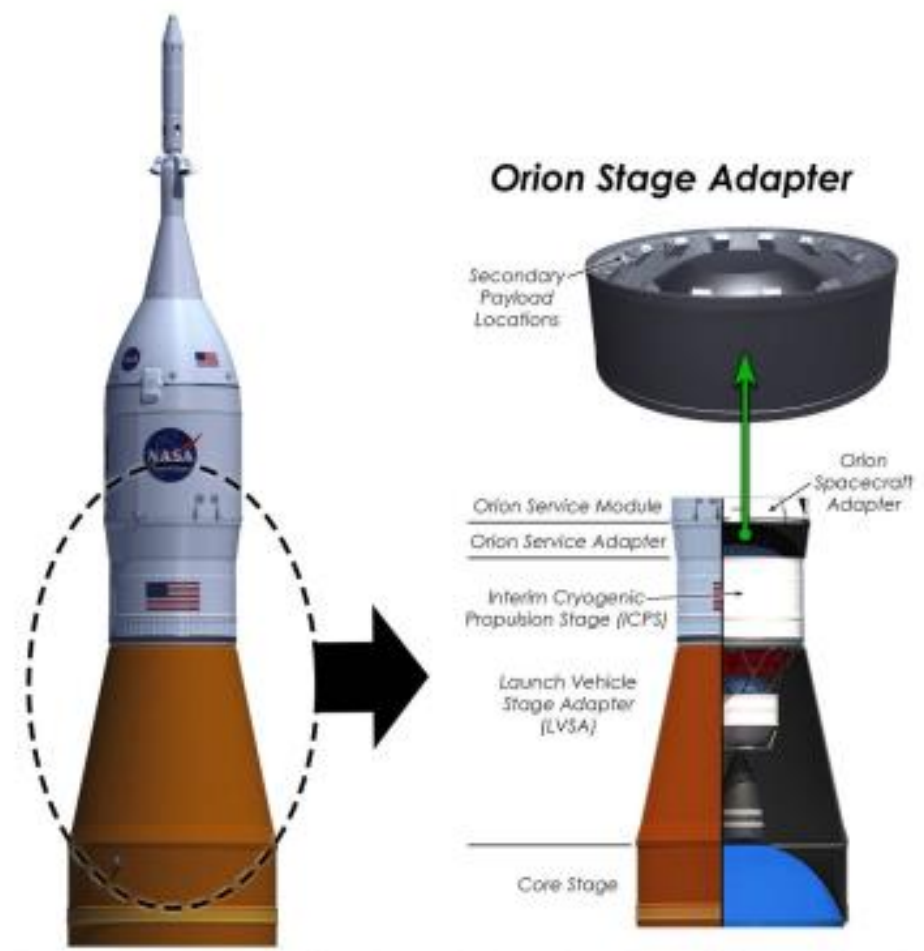

\section{Space Launch System (Block 1 Configuration)}

Figure 7. Cutaway view of the Spacecraft/Payload Elements of the Block 1 vehicle.

\section{B. Orion Stage Adapter (OSA)}

The second of two (2) adapters required to mate the Orion Crew Capsule to the SLS Launch Vehicle, the OSA attaches at the upper ring of the ICPS hydrogen tank and at the interface to the Orion Service Module (Figure 8). Approximate dimensions of the lightweight aluminum cone are $5.1 \mathrm{~m}(\sim 17 \mathrm{ft})$ and $5 \mathrm{~m}(\sim 16.5 \mathrm{ft})$ for the forward and aft circumferences respectively.

The OSA provides both a structural role as well as routing of communication cabling as with the LVSA. The lightweight panels are machined to engineering requirements incorporating, as with the LVSA, a lattice-like configuration, in this case utilizing an Iso-Grid to reduce weight while preserving required structural integrity. A key additional capability afforded by the OSA is the mounting of up to seventeen (17) secondary payloads as a secondary mission objective to enable cis-lunar scientific data collection opportunities. Additional detail regarding currently manifested secondary payloads and associated scientific objectives is covered in Section V.

A key benefit of using the ICPS is the geometric similarity to the DCSS that is employed by the ULA Delta IV Launch Vehicle. Specifically, when developing and manufacturing hardware for the Engineering Flight Test (EFT-1), launched in December 2014, the same OSA adapter design developed for SLS (and the corresponding induced loas), was able to be utilized for that flight test opportunity.

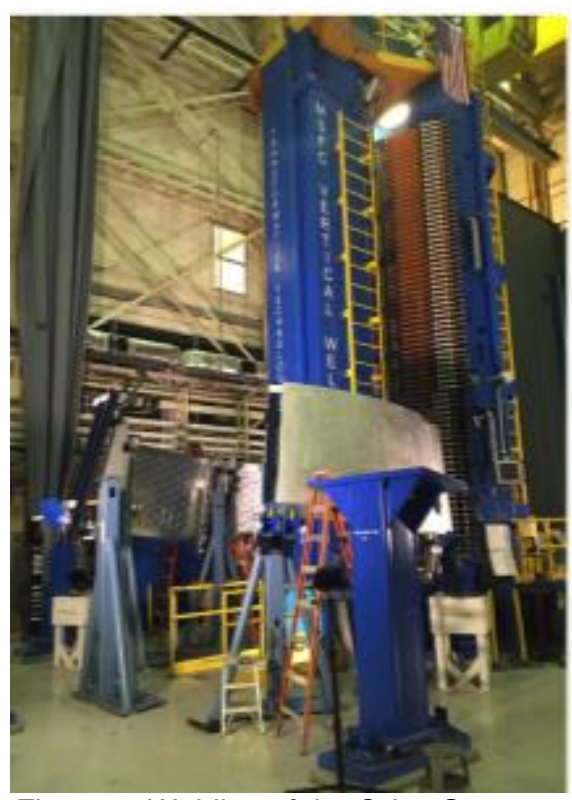

Figure 8. Welding of the Orion Stage Adapter at Marshall Space Flight Center. 
An additional cost savings was realized as a result of the STA article that was produced and tested for the EFT-1 mission. Following successful completion of that test, subjected to the lower Delta IV Launch Vehicle loads, the unit was placed into controlled storage at MSFC. Since it was, as previously mentioned, designed to SLS induced loads, the article was still available for the Integrated Structural Test in support of SLS and therefore no additional unit was required.

Work has subsequently been completed for the EM-1 flight article main structure with the composite diaphragm manufacturing underway.

\section{Interim Cryogenic Second Stage (ICPS)}

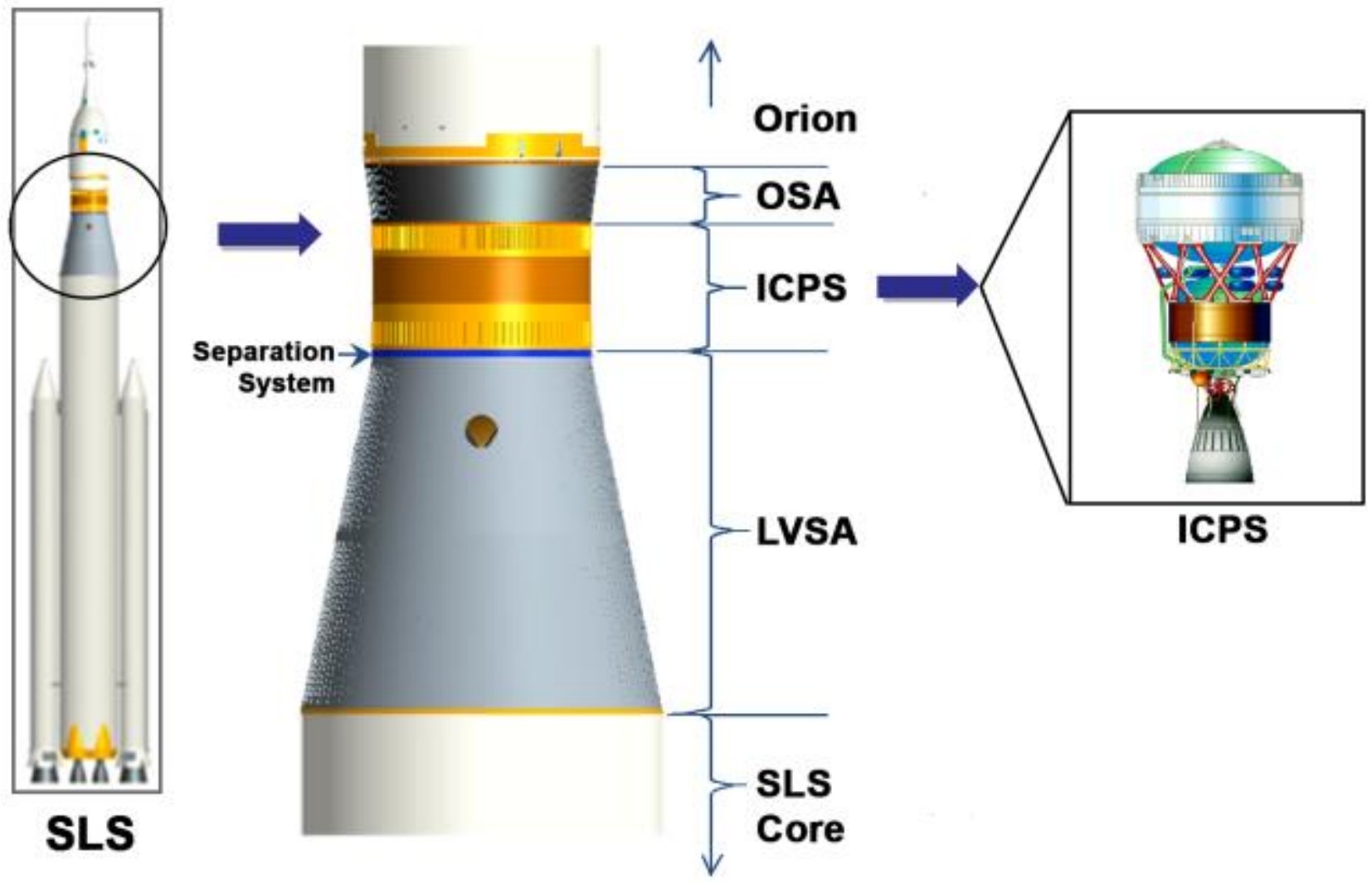

Nested inside the LVSA is the critical component necessary to put the Orion Crew capsule on the proper TransLunar Injection (TLI) trajectory for missions to the moon. As noted, the ICPS is a modified version of the DCSS currently in use by the ULA family of Launch Vehicles (Atlas \& Delta) with a demonstrated record of mission success. Utilization of existing hardware represents an efficient use of resources that allows an overall cost avoidance and ability to allocate funding to other areas of need.

Specifically, the modifications incorporated include lengthening the liquid hydrogen fuel tank for additional performance, additional hydrazine capacity for attitude control needs, and avionics tailored to SLS mission requirements.

Both the Structural Test Article and EM-1 Flight units are fabricated by ULA at their manufacturing facility located in Decatur Alabama. Major components such as the liquid oxygen and liquid hydrogen propellant tanks are manufactured at the facility, while the avionics components are built and delivered for installation from the ULA Facility in Denver, CO. The actual propulsion is generated by an Aerojet Rocketdyne RL-10B2 engine. 
Currently, the Structural Test Article required for integration with other SPIE hardware for the Integrated Structural Test has been completed and delivered to MSFC. (Figure 9)

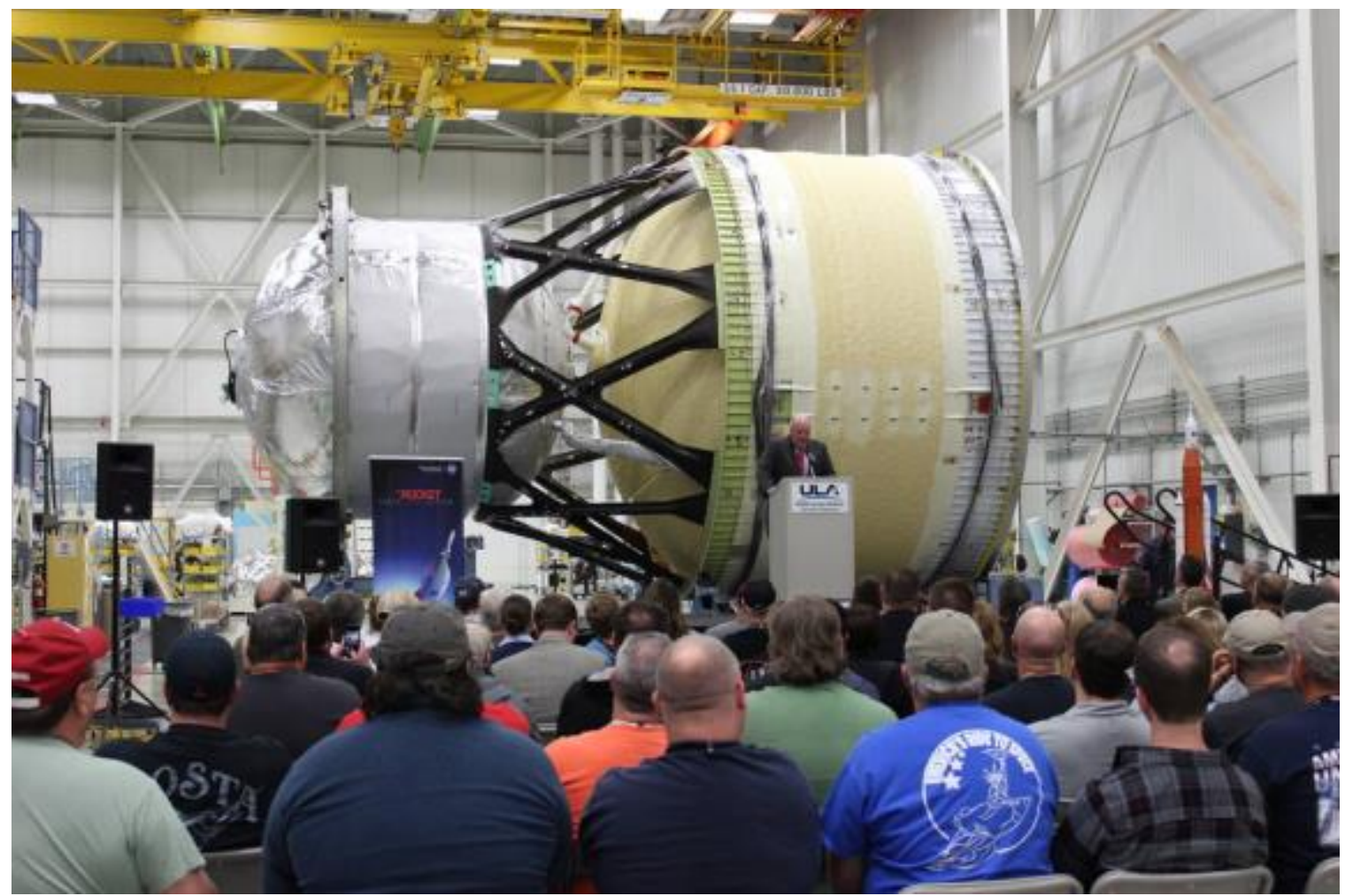

Figure 9. Event at United Launch Alliance in Decatur, AL marking completion of the ICPS test article.

\section{Integrated Structural Test (IST)}

Subsequent to the development and baselining of individual hardware component requirements and the associated design solutions, it is necessary to test the hardware as appropriate to validate that individual and integrated requirements are met. In addition, testing can both anchor and confirm that the analyses models developed and matured are sufficiently accurate for predicting hardware capability and associated safety factors/margins. These analyses are required products for successful completion of a Design Critical Review (DCR), the final Systems Engineering milestone to be conducted prior to entering the Flight Readiness Review process and ultimately proceeding to launch.

As noted, the SPIE Element is responsible for the management and integration of three (3) hardware components (LVSA, ICPS, and OSA). Each component is required to be structurally tested to assure compliance with levied structural requirements. However, the substantial costs associated with executing individual tests, coupled with the analytical uncertainty that would result from combining the results into an integrated model, led the Element to serve as the integrating test conductor for a combined structural test, assembling and conducting the test at MSFC. Each hardware Prime (TBE, Boeing/ULA, MSFC) is a Team member in the development of test requirements/parameters, test execution, and is provided test results for the noted model and hardware capability assessments.

Figure 10 illustrates the test configuration developed to implement and conduct the IST including required load rings and loadline actuators. In addition to hardware STA articles, structurally and dimensionally representative of flight articles, simulators representing the Orion and Core Stage Elements are included forward and aft respectively. It should be noted that the test stand structure employed to conduct the test is an adapted and modified stand that was originally used for cryogenic tank testing, resulting in additional and substantial cost savings. 
As stated previously, all component STA articles have completed manufacture, including the Orion and Core Stage simulators with test stand-specific activities on-plan and nearing completion. Stacking of the test articles into the stand commenced in mid-August, with the test start to begin in late 2016 and complete in early 2017.

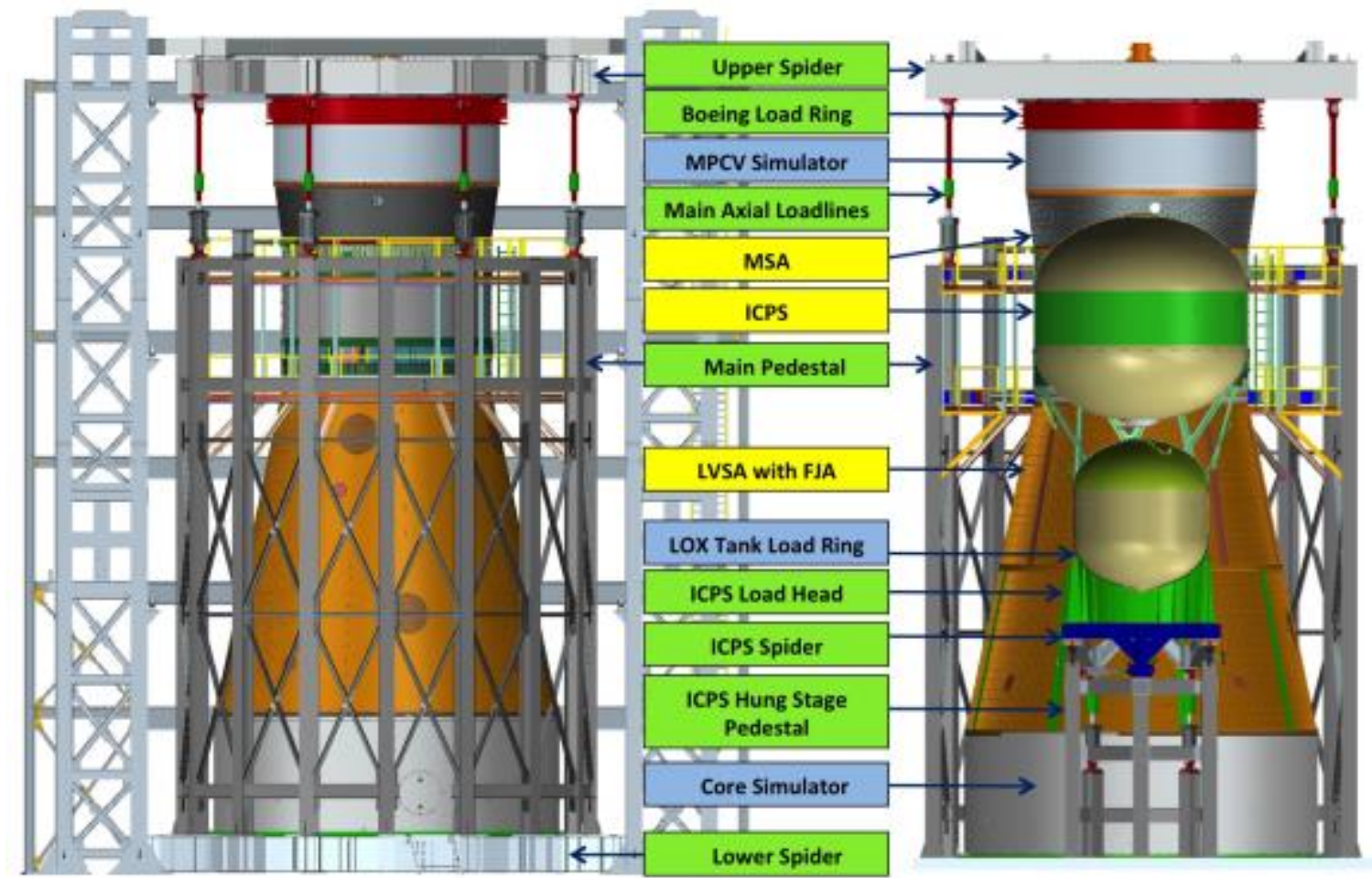

Figure 10. Arrangement of test articles for the Integrated Structural Test.

\section{Secondary Payloads}

While the most obvious mission profiles to benefit from SLS are those with requirements beyond the performance of current launch vehicles, SLS will also offer unique opportunities for smaller experiments in the form of secondary payload berths. Thirteen secondary payload locations will be available in the Orion-to-Stage Adapter in the initial SLS configuration, allowing payload deployment following Orion separation. The deployment berths are sized for "6U" CubeSats, and on EM-1 the spacecraft will be deployed into cislunar space following Orion's separate from the SLS Interim Cryogenic Propulsion Stage. Payloads in 6U class will be limited to $14 \mathrm{~kg}$ maximum mass. Concepts are still being reviewed for secondary payload accommodations on the evolved configurations of SLS. There is potential for these configurations to carry a larger class of secondary payload, which could also be deployed either into cislunar space with Orion or to another deep-space destination, accompanying a primary science payload.

CubeSat payloads on EM-1 will include both NASA research experiments and spacecraft developed by industry, international and potentially academia partners. The Human Exploration and Operations Mission Directorate (HEOMD) Advanced Exploration Systems (AES) Division was allocated five payload opportunities on the EM-1 mission. AES selected the first three payloads to fly on EM-1 at the same time the capability for accommodating Secondary Payloads on the SLS was being developed. Near Earth Asteroid (NEA) Scout is a 6U cubesat designed to rendezvous and characterize a candidate NEA. A solar sail, another innovation to be demonstrated in the cubesat class, will provide propulsion. Lunar Flashlight is the second AES payload planned for manifest on EM-1. It will use a green propellant system and will search for potential ice deposits in the Moon's permanently shadowed craters. 
Pulsed lasers will be used to illuminate the surface. Surface reflection will be measured by a spectrometer to distinguish water ices from regolith. The third payload being developed by AES is BioSentinel. The payload is a yeast radiation biosensor, planned to measure the effects of space radiation on Deoxyribonucleic Acid (DNA). This will be accomplished by entering into a heliocentric orbit, outside of the Van Allen belts, to expose the payload to a deep space radiation environment. Two additional payloads were selected for the EM-1 mission by AES from the Next Space Technologies for Exploration Partnerships (NextSTEP) Broad Agency Announcement (BAA). The payloads selected are Lunar Icecube, a collaboration with Morehead State University, and Skyfire, a partnership with Lockheed Martin. Lunar Icecube will prospect for water in ice, liquid, and vapor forms as well as other lunar volatiles from a low-perigee, highly inclined lunar orbit using a compact Infrared spectrometer. Skyfire is a technology demonstration mission that will perform a lunar flyby, collecting spectroscopy, and thermography data to address questions related to surface characterization, remote sensing, and site selection.

NASA's Space Technology Mission Directorate (STMD) was allocated three payload opportunities on the EM-1 mission. NASA's STMD is innovating, developing, testing, and flying hardware for use in NASA's future missions through the Centennial Challenges Program. The Centennial Challenges Program is NASA's flagship program for technology prize competitions (www.nasa.gov/challenges). The program directly engages the public, academia, and industry in open prize competitions to stimulate innovation in technologies that have benefit to NASA and the nation. STMD has released the CubeSat Lunar Challenge to foster innovations in small spacecraft propulsion and communications. There are two concurrent In-Space Competitions, the Lunar Derby and the Deep Space Derby. In the lunar Derby, there are prizes awarded for successfully achieving lunar orbit, downlinking the largest volume of error-free data and surviving the longest. In the Deep Space Derby ( $>4$ million $\mathrm{km})$, there are prizes awarded for farthest data transmission distance, largest volume of error-free data and longest duration of operability. Potential candidates for the three STMD opportunities on the EM-1 mission will compete in a series of four Ground Tournaments before final selection is made. Currently, two of the four Ground Tournaments have been completed. Final selection will be made in March of 2017.

The NASA Science Mission Directorate (SMD) was allocated two payload opportunities on the EM-1 mission. The NASA SMD issued an amendment to its annual Announcement of Opportunity (AO) in the Research Opportunities in Space and Earth Sciences-2014 (ROSES-2014) Solicitation NNH14ZDA001N-HTIDS Heliophysics Technology and Instrument Development for Science. Within this Amendment was the request for Cubesat proposals specific to the Exploration Mission 1 launch opportunity focusing on the Heliophysics science enabled through the unique deployment location and trajectory afforded though the planned EM-1 mission. The Cubesat Mission to Study Solar Particles (CuSP) payload was selected under this AO. CuSP will study the sources and acceleration mechanisms of solar and IP particles in near-Earth orbit, support space weather research by determining proton radiation levels during Solar Energetic Particle (SEP) events and identifying suprathermal properties that could help predict geomagnetic storms. A Small Innovative Missions for Planetary Exploration (SIMPLEx) NASA Research Announcement (NRA) was also released as part of the ROSES-2014 AO. The LunaHMap payload was selected from this NRA. The LunaH-Map objectives are to understand the quantity of H-bearing materials in lunar cold traps $(\sim 10 \mathrm{~km})$, determine the concentration of H-bearing materials with $1 \mathrm{~m}$ depth, and constrain the vertical distribution of H-bearing materials.

The final three payload opportunities for the EM-1 mission were allocated for NASA's international space agency counterparts. The flight opportunities are intended to benefit the international space agency and NASA as well as further the collective space exploration goals. A joint process with NASA and the international partners was employed to review, evaluate, and recommend the payloads to fly on EM-1. From that joint process three payloads were chosen: Omotenashi, (formerly SLS Launched Innovative Mission [SLSLIM]), ArgoMoon, and EQUilibriUm Lunar-Earth point 6U Spacecraft (EQUULEUS). ArgoMoon is sponsored by ESA/ASI and will fly-along with the ICPS on its disposal trajectory. The primary objectives are to perform proximity operations with the ICPS postdisposal, take external imagery of engineering and historical significance, and perform an optical communications demonstration. The EQUULEUS spacecraft sponsored by JAXA will fly to a libration orbit around the Earth-Moon L2 point and demonstrate trajectory control techniques within the Sun-Earth-Moon region for the first time by a nano spacecraft. The mission will also contribute to the future human exploration scenario by understanding the radiation environment in geospace and deep space, characterizing the flux of impacting meteors on the far side of the moon, and demonstrating the future deep space exploration scenario using the "deep space port" at Lagrange points. The Omotenashi mission sponsored by JAXA will land the smallest lunar lander to date on the lunar surface 
to demonstrate the feasibility of the hardware for distributed cooperative exploration system. Small landers will enable multi-point exploration, which is complimentary with large-scale human exploration. Once on the lunar surface, the Omotenashi spacecraft will observe the radiation and soil environments of the lunar surface by active radiation measurements and soil shear measurements.

\section{Future Evolution Of SLS}

The evolved configurations of SLS, including both the $105 \mathrm{t}$ Block 1B and the $130 \mathrm{t}$ Block 2, offer opportunities for launching co-manifested payloads and a new class of secondary payloads with the Orion crew vehicle, and also offer the capability to carry primarily payloads within 8.4 - or $10-\mathrm{m}$ payload fairings, larger than any contemporary launch vehicle, delivering unmatched mass-lift capability, payload volume, and C3.

As early as the second launch of SLS, Exploration Mission-2, the vehicle will be augmented with a low-thrust dual-use Exploration Upper Stage (EUS), providing both ascent and in-space propulsion capabilities. This stage, which is working toward a preliminary design review in late 2016, will upgrade SLS to a performance of $105 \mathrm{t}$ to LEO, and create a configuration that will serve as a workhorse for "Proving Ground" missions in cislunar space that will pave the way for further exploration. From there, additional upgrades, including enhancements to the RS-25 engines and upgraded boosters will ultimately evolve SLS to a configuration capable of delivering more than 130 metric tons to LEO, the capability identified as necessary for human missions to Mars.

Early research has also been conducted into options for larger 8.4- and 10-m fairings, with which SLS will potentially offer payload volumes of 1,200 and 1,800 cubic meters, respectively. With a 10-m fairing, the vehicle will be able to offer payload volumes five times greater than currently available. In addition to those traditional classes of payload fairings, the Space Launch System offers additional unique payload capabilities, including launch of a co-manifested payload along with the Orion spacecraft or delivery of secondary payloads to lunar or planetary trajectories. A co-manifested payload, with a volume of up to 400 cubic meters, could be placed within the Universal Stage Adapter connecting the Exploration Upper Stage to the Orion stack, and could be used, among other purposes, to deliver multi-ton payloads to a destination alongside Orion, allowing, for example, deployment of Orion and a habitat with crew to cislunar space with a single launch.

Work is already underway on future configurations of the vehicle. System requirement and design reviews for the Exploration Upper Stage were completed in late 2015, and the Program is working toward a preliminary design review on the stage in late 2016. Boeing will be the contractor for the EUS, with Aerojet Rocketdyne providing the RL-10-C3 engines. NASA's Glenn Research Center will manage the Universal Stage Adapter, and is preparing for a request for proposal for the USA in late 2016. Early risk reduction and engineering demonstration work has also been conducted for concepts related to future booster upgrades. 


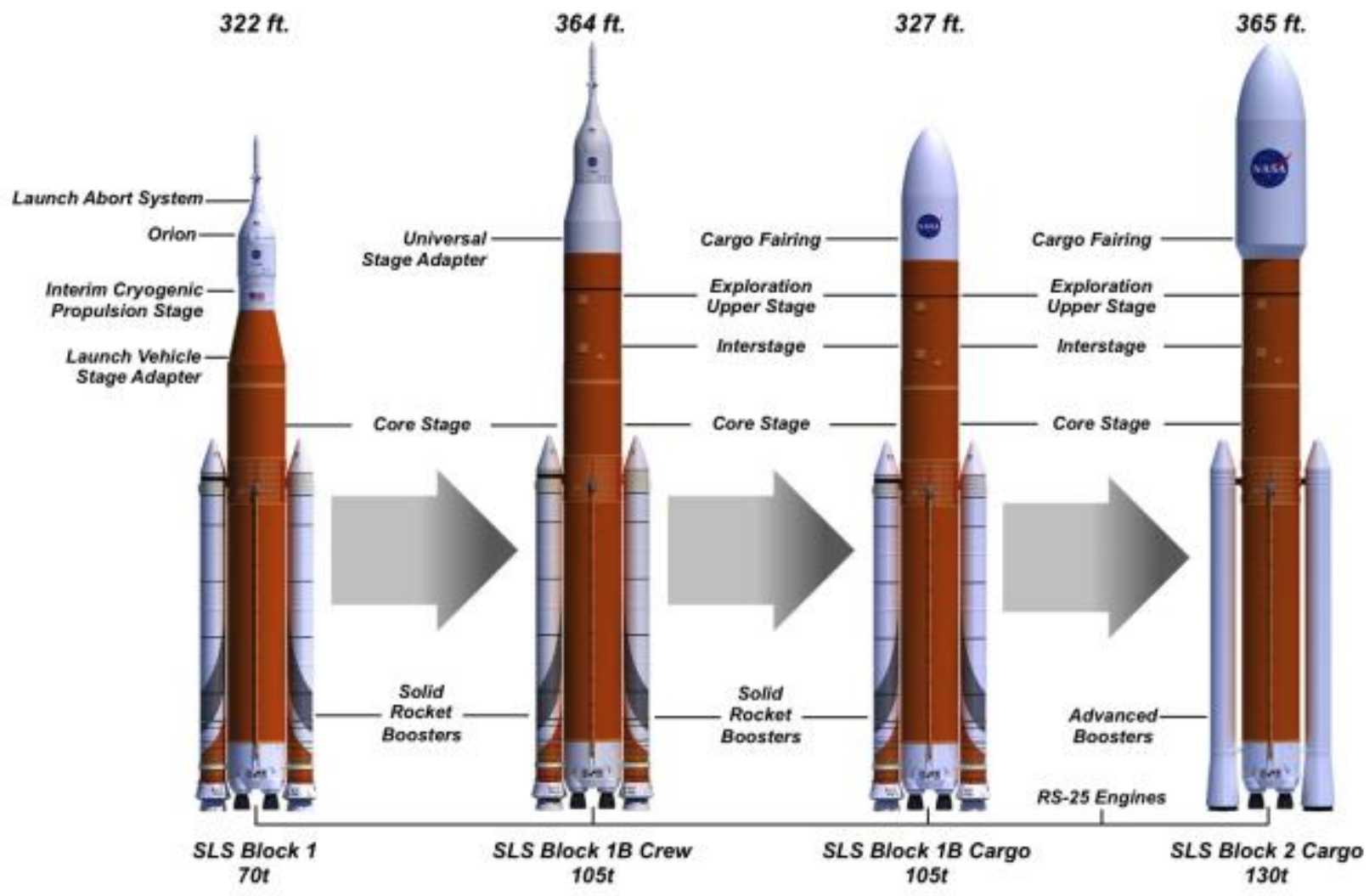

Figure 11. SLS Evolutionary Path

\section{SLS Capabilities and Utilization}

The capabilities of the Space Launch System provide not only the capability to conduct human exploration of deep space, but also game-changing benefits for a range of promising space science missions. Three major interrelated areas have been identified in which SLS offers unique benefits that make possible new missions or mission profiles - unrivaled mass-lift capability, payload volume capacity, and departure energy. Taking advantage of these benefits allows spacecraft designers and mission planners to change fundamental assumptions about spacecraft and mission design, as these areas offer the potential for numerous benefits:

- Less-complex payload design and miniaturization needed to fit in fairings, leading to increased design simplicity.

- Decreased launches for in-space assembly, resulting in reduced risk.

- Less folding/deployment complexity, leading to increased mission reliability and confidence.

- High-energy orbit and shorter trip times, leading too less expensive mission operations and reduced exposure to the space environment.

- Increased lift capacity and payload margin, resulting in less risk. 
The high mass-lift capability of SLS is vital to human deep-space exploration, allowing the launch of the Orion crew vehicle and other exploration systems to a staging location in the lunar vicinity, or directly into deep space. Not only will SLS be able to launch large systems in a cargo configuration, it will be able to launch payloads, such as smaller habitat modules, co-manifested with Orion within the Universal Stage Adapter connecting the crew vehicle with the rocket's upper stage. This adapter offers greater payload volume than any contemporary EELV payload fairing, opening opportunities for singlelaunch of longer-duration deep-space stays.

SLS' characteristic energy (C3) offers reduced mission transit time, thereby reducing power requirements as well as the amount of time that scientific instruments are exposed to space. While commercial launchers will continue to serve as the workhorse for many of NASA's science missions, those spacecraft often have to make multiple gravityassist maneuvers around inner planets before reaching the velocity needed to reach the outer planets. These maneuvers increase mission times by years and increase risk to onboard instruments because of the extended time in the space environment and the range of conditions to which they require exposure.

SLS utilization is currently being considered for NASA's proposed Europa Multiple Flyby Mission, which would provide an unprecedented look at the icy Jovian moon, believed to hold a subsurface ocean with more than twice the quantity of water on Earth, and investigate its potential habitability.

While launch on an Atlas V 551 EELV-baseline vehicle could require a Venus-Earth-Earth gravitational assist trajectory requiring 7 to 8 years, launch on SLS would enable a direct transit to the Jovian system in less than three years, providing far earlier science return and reduced operational costs, among other benefits (Figure 12). With consideration currently ongoing of a follow-up Europa lander mission, the earlier science return could allow use of data from the flyby mission to inform the lander mission, without a substantial delay to the latter.

Europa mission analysis also serves as a test case for how SLS could benefit outer-planet exploration. One of the major benefits to the science community from the Mars Program has been the ability to learn from one mission and use that knowledge when formulating a near-term future investigation. The paradigm for outer planet exploration has necessitated very long cruise times, which, among other things, make it impossible to have a rapid turnaround in penetrating the mysteries that the "ocean world" icy moons of the outer planets possess. The availability of the SLS breaks this model, and allows for significant transit-time reduction.

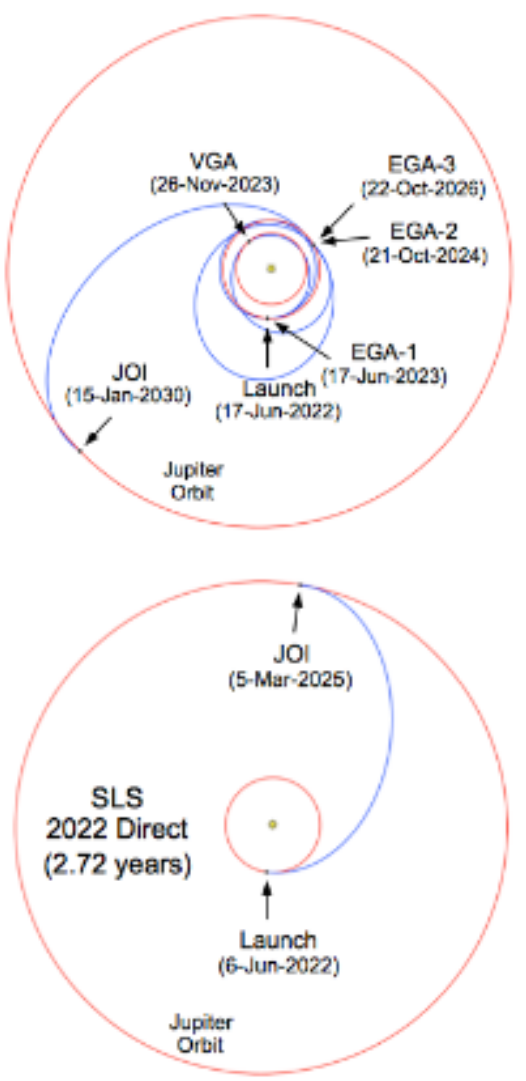

Figure 12. Gravitational-assist trajectory to Europa enabled by current EELVs (top) versus direct trajectory enabled by SLS (bottom).

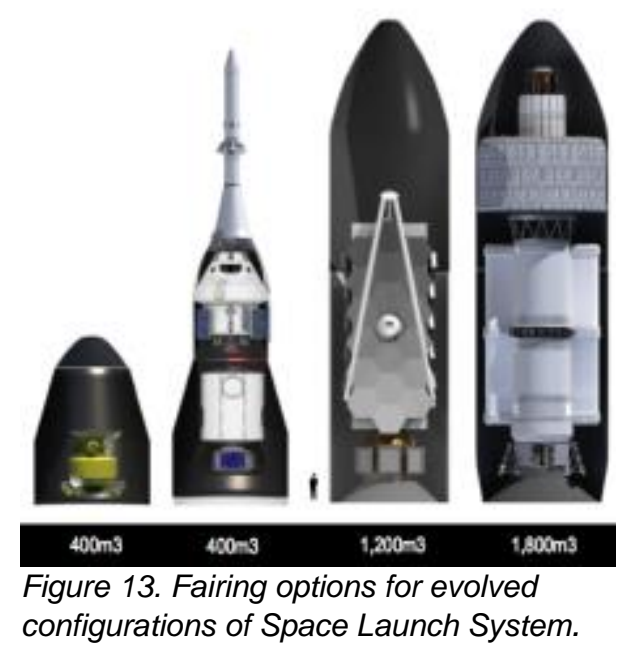


In the area of payload volume capacity, a large-aperture space telescope offers a good case study. Concept evaluation has demonstrated potential benefits of a large 8.4- or 10-m SLS payload fairing for the science community. Such a fairing would enable the launch of a large aperture (potentially 16-m class) telescope that would be able to make ultra-high-contrast spectroscopic observations of exoplanets. Such a capability would address a need identified in the 2013 NASA astrophysics roadmap, "Enduring Quests, Daring Visions." Concept evaluations of such a project have also identified opportunities for further collaborations between science and human exploration systems in the form of assembly and servicing of an observatory in deep space.

\section{Conclusion}

Substantial progress has been made on the development and manufacture of the initial $70 \mathrm{t}$ Block 1 configuration of SLS, and its first launch will be a significant step in demonstrating the super-heavy-lift capability needed for human exploration of deep space. With the evolution of SLS beginning with its second launch, this vehicle will return humans to deep space for the first time in decades, beginning a series of exploration missions that will lead to Mars and other destinations that will reveal an unprecedented wealth of knowledge about our solar system and universe. 